\title{
Magnetic field dependence of the blob dynamics in the edge of ASDEX Upgrade L-mode plasmas
}

\author{
G. Birkenmeier, ${ }^{1, *}$ F. M. Laggner, ${ }^{2}$ M. Willensdorfer,${ }^{2}$ T. Kobayashi,${ }^{3}$ P. Manz,${ }^{1}$ E. Wolfrum,${ }^{1}$ \\ D. Carralero, ${ }^{1}$ R. Fischer, ${ }^{1}$ B. Sieglin, ${ }^{1}$ G. Fuchert,${ }^{4}$ U. Stroth,${ }^{1,5}$ and the ASDEX Upgrade Team \\ ${ }^{1}$ Max Planck Institute for Plasma Physics, Boltzmannstr. 2, 85748 Garching, Germany \\ ${ }^{2}$ Institute of Applied Physics, Vienna University of Technology, \\ Association EURATOM-ÖAW, 1040 Vienna, Austria \\ ${ }^{3}$ Interdisciplinary Graduate School of Engineering Sciences, Kyushu University, Kasuga 816-8580, Japan \\ ${ }^{4}$ Institut für Grenzflächenverfahrenstechnik und Plasmatechnologie, \\ University of Stuttgart, 70569 Stuttgart, Germany \\ ${ }^{5}$ Physik-Department E28, Technische Universität München, 85747 Garching
}

\begin{abstract}
The magnetic field dependence of intermittently expelled density filaments (blobs) is investigated in the scrape-off layer of ASDEX Upgrade low confinement (L-mode) plasmas. It is demonstrated that lithium beam emission spectroscopy can be used to determine the frequency, radial size and velocity of the blobs. The measured radial blob sizes depend only weakly on magnetic field $B$. Normalizing the blob sizes to the drift parameter $\rho_{s} \propto B^{-1}$ results in a large variation beneficial for a quantitative comparison with theoretical blob scaling laws. The blob velocity scales inversely proportional to the square of the blob size in agreement with analytic models for blobs in the sheathconnected regime. The measurements point towards an influence of finite ion temperature on radial blob transport.
\end{abstract}

PACS numbers: 52.25.Xz, 52.25.Fi, 52.35.Ra, 52.55.Fa

\section{INTRODUCTION}

In the scrape-off layer (SOL) of toroidal magnetic fusion devices, intermittently expelled density filaments generated in the plasma edge appear in practically all discharge regimes. Advected by the magnetic field line curvature driven $E \times B$ drift, they transport a significant fraction of heat and particles perpendicular to the magnetic field towards the wall. The estimation of this convective contribution to SOL transport is essential for a safe and controlled operation of future fusion devices [1]. Hence, the physics of these filaments, which are also called blobs according to their shape in the twodimensional plane perpendicular to the magnetic field, are extensively studied experimentally $[2,3]$ and theoretically [4].

For blobs in the low confinement discharge regime (Lmode), several analytical velocity scaling laws have been derived which relate the radial velocity of a blob $v_{r}$ to its size $a$ perpendicular to the magnetic field. Depending on the collisionality, neutral density and magnetic field line geometry, different regimes for the velocity scaling have been proposed [5-9]. Although considerable high ion temperatures prevail in the far SOL of fusion devices [10], the influence of finite ion temperatures has mostly been neglected for the sake of simplicity in the derivations. Recently published velocity scaling laws [11] derived from a drift-interchange-Alfvén fluid model take warm ion effects into account.

The main purpose of our investigations presented here

\footnotetext{
* gregor.birkenmeier@ipp.mpg.de
}

is to compare measured blob velocities with different theoretical scaling laws in order to validate the scalings and indirectly investigate the contribution of warm ions on blob transport. For this purpose, the blob velocities and sizes were measured in the SOL of ASDEX Upgrade in low density L-mode discharges using lithium beam emission spectroscopy (Li-BES) [12]. The measurements were done for a wide range of the magnetic field strength $B$ in order to achieve a maximum variation of the blob size $a$ which should depend on $B$ due to its relation $a \propto \rho_{s}^{4 / 5}$ [4] to the drift scale $\rho_{s}=\sqrt{T_{e} m_{i}} / e B$ with electron temperature $T_{e}$, ion mass $m_{i}$ and elementary charge $e$.

For the experimental determination of the blob velocity and the size, the Li-BES system at ASDEX Upgrade is well suited due to the simultaneous acquisition of fluctuation data along the lithium beam path from slightly inside the separatrix up to the wall covering the entire SOL. In contrast to reciprocating probes, the Li-BES system can measure over the entire discharge duration. While gas puff imaging (GPI) systems measure an intensity depending on a combination of electron density and temperature [13], the Li-BES signal is dominantly sensitive to electron density, and the poloidal and toroidal localization of the beam emission $(\sim 1 \mathrm{~cm})$ is typically smaller than the gas cloud width relevant for GPI. This makes the interpretation of Li-BES data easier compared with other optical diagnostics. Nevertheless, the Li-BES fluctuation data should always be validated with radiativecollisional modeling in order to avoid misinterpretations due to the finite lifetime of the Li I state which leads to a smearing of the signal along the beam path [12].

In the remaining parts of this paper, the experimental setup (Section II) and the conditional averaging method applied to Li-BES data for the determination of blob size 
and velocity (Section III) are presented. In Section IV, the properties of blobs depending on the magnetic field $B$ are shown and compared with different blob scaling laws in Section V. The results will be summarized in Section VI.

\section{EXPERIMENTAL SETUP AND DISCHARGE PARAMETERS}

The experiments for this study have been performed at the medium size tokamak ASDEX Upgrade in ohmically heated L-mode discharges in lower single null configuration. In order to achieve a largest possible variation of the blob size $a$ which should also result in a large variation of the blob velocity $v_{r}$ according to scaling formulas, the full accessible range of the toroidal magnetic field from $B_{t}=-1.4$ to $-3.2 \mathrm{~T}$ was used on a shot to shot basis. The minus sign indicates the magnetic field vector pointing clockwise in opposite direction of the plasma current $I_{p}$. Two magnetic field scans have been performed, one with constant toroidal current $I_{p}=600 \mathrm{kA}$ and another one with constant edge safety factor $q_{95} \approx 5$. While the first scan at constant $I_{p}$ is associated with a variation of the parallel connection length $L_{||}$of the magnetic field line, the second scan kept $L_{\|}$constant. This allows to decouple the impact of $L_{\|}$and $B_{t}$ both of which enter sensitively into most of the blob scaling formulas (see Section V). Selected discharge parameters used in the two $B_{t}$ scans are summarized in Table I.

The density of all discharges was kept constant via density feedback control at an edge density value of $n_{e, 95} \approx 2.0 \cdot 10^{19} \mathrm{~m}^{-3}$. The density profiles were measured with the standard lithium beam diagnostics ("LiIXS", see Ref. [12]). A probabilistic profile reconstruction algorithm [14] allows to calculate the most probable density profile for a given measured Li I emission profile by means of a collisional-radiative model. The resulting density profiles are shown in Fig. 1. They are the most accurate profiles compared to other diagnostics in the region from the pedestal top to the main chamber wall at the outer midplane. The density profiles within the data set at constant $I_{p}$ (Fig. 1(a)) are similar to each other. Likewise the profiles in the data set at constant $q_{95}$ (Fig. 1(b)) are similar to each other and also similar to the profiles at constant $I_{p}$ indicating reproducible conditions despite the large variation of $B_{t}$.

The electron temperature was measured using Thomson scattering yielding for all discharges values in the range of $T_{e}=12 \pm 8 \mathrm{eV}$ at the normalized poloidal flux coordinate position $\rho_{\text {pol }}=1.044$ which was chosen as reference for the analysis presented in Section III due to the highest sensitivity of the Li-BES system for blobs at this radial position [12]. The electron temperatures from Thomson scattering agree with $T_{e}$ from Langmuir probe measurements in comparable discharges [15].

The Li-BES diagnostics at ASDEX Upgrade was recently upgraded with a new bundle of lines of sight (LOS)
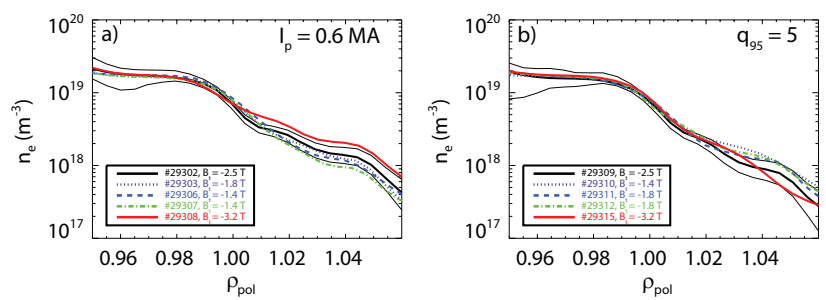

FIG. 1: Radial electron density profiles at the plasma edge and SOL in logarithmic representation for different toroidal magnetic fields $B_{t}$ at constant plasma current $I_{p}$ (a) and edge safety factor $q_{95}$ (b). The black thin lines mark the error bars for \#29302 which are representative for all profiles.

and a new optical head optimized for turbulence measurements in terms of an increased photon flux and a higher data acquisition rate of $200 \mathrm{kHz}$ corresponding to a time resolution of $5 \mu \mathrm{s}$. Details of the upgrade and the arrangement of the LOS can be found in Ref. [12]. For blob studies, only the 15 outermost channels named MSIG(1) to MSIG(15) around the separatrix $\left(\rho_{\text {pol }}=1\right)$ were used corresponding to a radial range of $\rho_{\text {pol }}=1.08$ to 0.97 . A single observation volume had a horizontal width of $5 \mathrm{~mm}$ and a distance of $6 \mathrm{~mm}$ to the adjacent volumes. The LOS were arranged horizontally $31.8 \mathrm{~cm}$ above the equatorial mid plane of the vessel allowing for size and velocity measurements mainly into radial direction (the poloidal inclination angle between the radial LOS array and the radial direction was smaller than 20 degree).

Due to collisions of the lithium beam neutrals with plasma particles (electrons, main and impurity ions), the $\mathrm{Li}_{2 \mathrm{p}}$ state is excited. The $\mathrm{Li}_{2 \mathrm{p}-2 \mathrm{~s}}$ line emission is mainly determined by the electron density $n_{e}$ and depends only weakly on electron or ion temperatures [16]. The line emission from the observation volumes was detected by means of photomultipliers and optical filters. Measuring the beam intensity in a neutral gas puff after every discharge, the relative variations of the photomultipliers were compensated, and in order to get rid of spurious background light from impurity lines, the beam was periodically (every $80 \mathrm{~ms}$ ) switched off for $24 \mathrm{~ms}$ by a fast extraction chopper [17] for background subtraction.

\section{BLOB DETECTION WITH LI-BES}

The $\mathrm{Li}_{2 \mathrm{p}-2 \mathrm{~s}}$ line emission signals were evaluated for all discharges listed in Tab. I for a time interval of 300 ms. Due to a large contribution of photon noise at high frequencies [12], the data was filtered with a low-pass Butterworth filter with a cutoff frequency of $20 \mathrm{kHz}$. Examples of these raw data are shown in Fig. 2. The upper row shows color coded zero-mean line emission signals $\delta I$ arranged along the coordinate $x_{s}=R-R_{\text {sep }}$ vs. time, with $R_{\text {sep }}$ the separatrix position and $R$ the major radius along the horizontal beam path. $x_{s}=0 \mathrm{~cm}$ corresponds 


$\left.\begin{array}{|c|c|c|c|c|c|c|c|}\hline B_{t}(\mathrm{~T}) & I_{p}(\mathrm{MA}) & q_{95} & L_{\|}(\mathrm{m}) & f_{G} & \text { discharge number } & \text { analyzed time interval }(\mathrm{s}) \\ \hline-2.5 & 0.6 & 6.85 & 14.99 & 0.35 & \# 29302 & 2.9,3.3 \\ -1.8 & 0.6 & 4.96 & 10.80 & 0.35 & \# 29303 & 2.9,3.3 . \\ -1.4 & 0.6 & 3.85 & 9.00 & 0.37 & \# 29306 & 2.9,3.3 . \\ -1.4 & 0.6 & 3.85 & 9.00 & 0.35 & \# 29307 & 2.9,3.3 . \\ -3.2 & 0.6 & 8.72 & 18.90 & 0.35 & \# 29308 & 2.9,3.3 . \\ \hline-2.5 & 0.83 & 4.90 & 10.90 & 0.25 & \# 29309 & 2.9,3.3 . \\ -1.4 & 0.47 & 5.00 & 11.50 & 0.45 & \# 29310 & 2.9,3.3 . \\ -1.8 & 0.6 & 4.94 & 10.90 & 0.35 & \# 29311 & 2.9,3.3 . \\ -1.8 & 0.6 & 4.96 & 11.10 & 0.36 & \# 29312 & 2.9,3.3 . \\ -3.2 & 1.07 & 4.96 & 10.80 & 0.22 & \# 29315 & 2.6,3.0]\end{array}\right\} I_{p}=$ const

TABLE I: Toroidal magnetic field $B_{t}$, plasma current $I_{p}$, edge safety factor $q_{95}$, parallel connection length $L_{||}$at $\rho_{p o l}=1.044$ from magnetic field line tracing, Greenwald fraction $f_{G}$, discharge number and considered time interval of the analyzed discharges.

to the separatrix position and $x_{s}>0$ indicate the SOL (red color corresponds to high intensity). In this representation, positive density blobs appear as vertically (=radially) elongated structures. At low magnetic field (Fig. 2(a)), many "thin", i.e. short-living, blobs are observed. Their radial extent is limited to a few centimeter around the separatrix. At medium fields (Fig. $2(\mathrm{~b}))$, more and more large events appear between the smaller ones. Their lifetime is longer and also the radial extent seems to be increased compared to the "thin" blobs. At high magnetic field (Fig. 2(c)), the dynamics is dominated by the large events. They appear tilted in the $x_{s}$-t-plane indicating a radial velocity in the range of about $100 \mathrm{~m} / \mathrm{s}$. This agrees with the picture that blobs are generated in the separatrix region and are expelled radially into the scrape-off layer [2].

In the signals MSIG(6) and $\operatorname{MSIG}(8)$, exemplarily shown in the lower row of Fig. 2, the same dynamics with a trend to larger events at higher magnetic field can be found. For these time traces the temporal average was not subtracted, thus one can estimate the relative amplitude of the blob with respect to the average intensity. For channels in the far SOL, the relative blob amplitude determined from the line emission intensity is proportional to the relative density amplitude, i.e. $\delta I / I \propto \delta n / n$ [12]. Taking this into consideration, we conclude from the time traces in Fig. 2(b), (d) and (f) that the blobs are not expelled into a SOL vacuum. For all plasma conditions, they propagate into a periphery of a background of nonzero density - at least within the temporal resolution of the Li-BES system $(5 \mu \mathrm{s})$.

In order to evaluate the radial size and velocity of the blobs, a conditional average technique [18] was applied to zero-mean, low-pass filtered Li-BES data. The cut-off frequency of the filter was $20 \mathrm{kHz}$. Channel MSIG(6) corresponding to a radial position at $\rho_{\text {pol }} \approx 1.044$ in the far SOL was chosen as reference. Every event in the time trace of channel MSIG(6) which surpassed the threshold of 2.5 standard deviations $\sigma$ (green line in the lower row of Fig. 2) was treated as a blob and used for the conditional averaging procedure. The averaged time windows around the detected blobs had a length of $500 \mu \mathrm{s}$. The conditional averaging procedure resulted in a statistically representative spatial-temporal picture $I\left(x_{s}, \Delta t\right)$ of the Li-BES signal shown in Fig. 3(a). $x_{s}$ is, as in Fig.
2 , the beam coordinate, and $\Delta t$ the time shift relative to the time point where the rising edge of the blob surpassed the $2.5 \sigma$-threshold. For the averaging, data from the beam-on phase during the time interval $t \in[2.9,3.3] \mathrm{s}$ in discharge \#29302 was used. The color coded intensity shows clearly a localized structure of high intensity (red color) with a spatial width (half width at half maximum, $\mathrm{HWHM})$ at $\Delta t=0$ of $\delta x=1.79 \mathrm{~cm}$. The temporal extension of the blob $\tau_{\text {blob }}$ evaluated as the full width at half maximum (FWHM) of $I\left(x_{s}, \Delta t\right)$ at the reference channel MSIG(6) corresponds to the time span a blob appears in front of our detector. This self-correlation time $\tau_{\text {blob }}$ is related to the lifetime of the blob $\tau_{\text {life }}$ but not identical since a blob typically exists longer than $\tau_{\text {blob }}$ and moves away from our detection region during its lifetime $\tau_{\text {life }}$. $\tau_{\text {blob }}$ depends on the chosen channel and amounts in the considered case to about $120 \mu \mathrm{s}$. This is far above the effective time resolution of $50 \mu$ s set by the low-pass filter applied to the raw data prior to the conditional averaging. As already observed in the raw data (Fig. 2, upper row), the contour areas of the highest intensities of $I\left(x_{s}, \Delta t\right)$ (e.g. the yellow and red contours) are tilted with an increased distance to the separatrix for later time points indicating a propagation. In order to quantify this radial propagation, we define a center-of-mass coordinate

$$
\begin{aligned}
X_{c} & =\frac{1}{Q} \int x_{s} I\left(x_{s}, \Delta t\right) d x_{s} \text { with } \\
Q & =\int I\left(x_{s}, \Delta t\right) d x_{s} .
\end{aligned}
$$

The bounds of integration cover the 15 outermost channels, and only positive values of $I\left(x_{s}, \Delta t\right)$ were taken into account in order to exclude negative events (holes). This way, we get an averaged blob position $X_{C}$ at every time point $\Delta t$ as shown for different snapshots of $I\left(x_{s}, \Delta t\right)$ in Fig. 3(b) (vertical lines). The temporal evolution of $X_{C}(\Delta t)$ as indicated in Fig. 3(c) (solid line) is a continuous and smooth trajectory allowing for linear fitting (dotted line) in order to determine the average radial blob velocity $v_{\mathrm{r} \text {,mean }}$ from its slope. The temporal derivative of the trajectory $X_{C}(\Delta t)$ also allows for the determination of a maximum velocity

$$
v_{\mathrm{r}, \max }=\max \left\{\frac{\mathrm{d} X_{C}(\Delta t)}{\mathrm{d} \Delta t}\right\},
$$



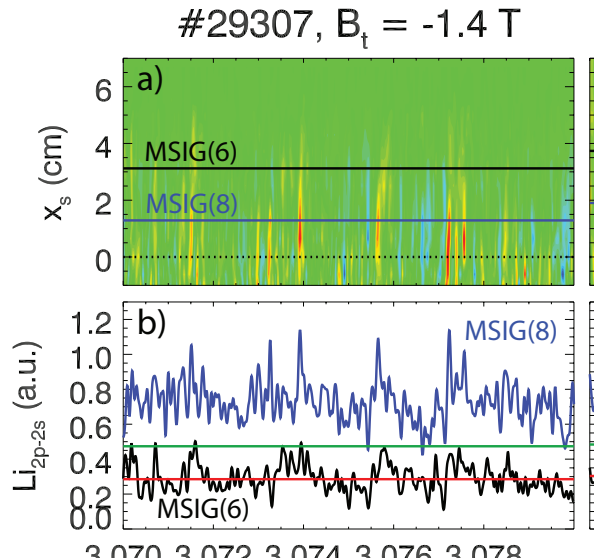

$\mathrm{t}(\mathrm{s})$
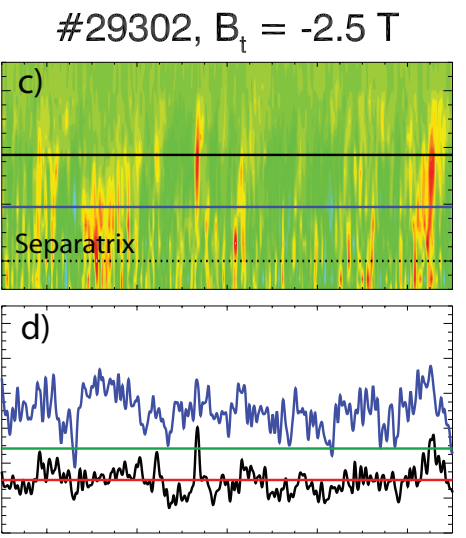

3.0763 .0783 .0803 .0823 .084

$\mathrm{t}(\mathrm{s})$
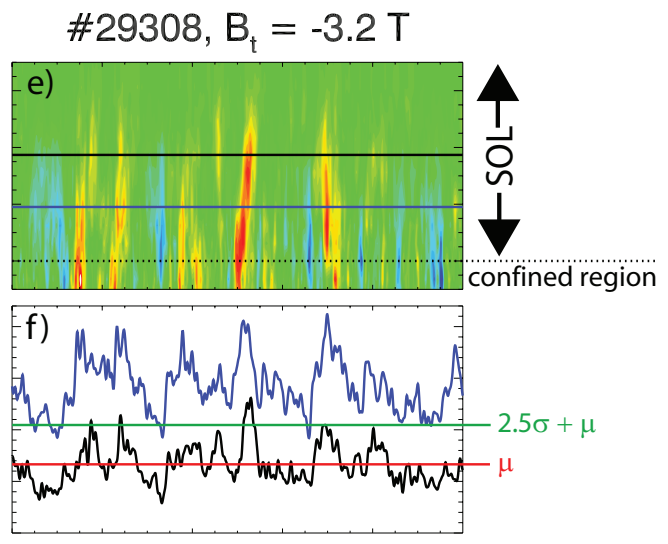

3.0723 .0743 .0763 .078

$\mathrm{t}(\mathrm{s})$

FIG. 2: Raw data from Li-BES at $B_{t}=-1.4 \mathrm{~T}$ (left), $B_{t}=-2.5 \mathrm{~T}$ (middle), and $B_{t}=-3.2 \mathrm{~T}$ (right). The upper row shows the intensity of the Li-BES signal (mean subtracted) for different radial distance to the separatrix $x_{s}$ in the SOL (red color corresponds to higher intensity) vs. time. Solid lines in the upper row indicate the radial positions of two selected signals MSIG(6) (black) and MSIG(8) (blue) shown in the lower row. The red line indicates the temporal mean $\mu$ and the green line the $2.5 \sigma$ threshold value for conditional averaging of the lower time series.

which is typically found around $\Delta t=0$. Due to the radial range of $\approx 9 \mathrm{~cm}$ given by the 15 considered LOS and the fact that the blob can vanish or appear within a minimum time of $12.5 \mu \mathrm{s}$ (corresponding to a quarter period at the given filter frequency of $20 \mathrm{kHz}$ ) the maximum detectable velocity amounts to $7.2 \mathrm{~km} / \mathrm{s}$.

The two definitions of blob velocities, $v_{\mathrm{r}, \text { mean }}$ and $v_{\mathrm{r}, \max }$, provide a lower and upper limit of possible velocities. This way we get useful range of velocity for the comparison with theoretical velocity scaling laws.

From the conditional averaging we obtain the selfcorrelation time $\tau_{\text {blob }}$, the average radial velocity $v_{\mathrm{r}, \text { mean }}$, the maximum radial velocity $v_{\mathrm{r}, \max }$, the width $\delta x$ and the relative amplitude $\delta I / I$ of the blob as they appear in the line emission signal of the Li-BES diagnostics. In order to compare these quantities with theoretical scalings, we must translate them into quantities in terms of density. We assume that the temporal behavior of the line emission is identical to the dynamics of the density evolution and use $\tau_{\text {blob }}$ and $v_{r}$ as characteristics for the density dynamics.

However, this is not possible for $\delta x$ and $\delta I / I$ due to the finite lifetime of the $\mathrm{Li}_{2 \mathrm{p}}$ state which leads to a smearing of the blob in the line emission response [12]. Thus, the blobs appear to be larger in the line emission than they actually are. This smearing also changes the relative line emission response amplitude $\delta I / I$ with respect to the initial relative blob amplitude $\delta n / n$.

In order to translate the original radial blob width $a$ defined as the HWHM of the density perturbation into the line intensity response width $\delta x$, dedicated numerical studies considering the radiative-collisional processes involved in the $\mathrm{Li}_{2 \mathrm{p}-2 \mathrm{~s}}$ line emission have been undertaken. The basic concept of this study was to put an artificial Gaussian density perturbation with a HWHM of $a$ and
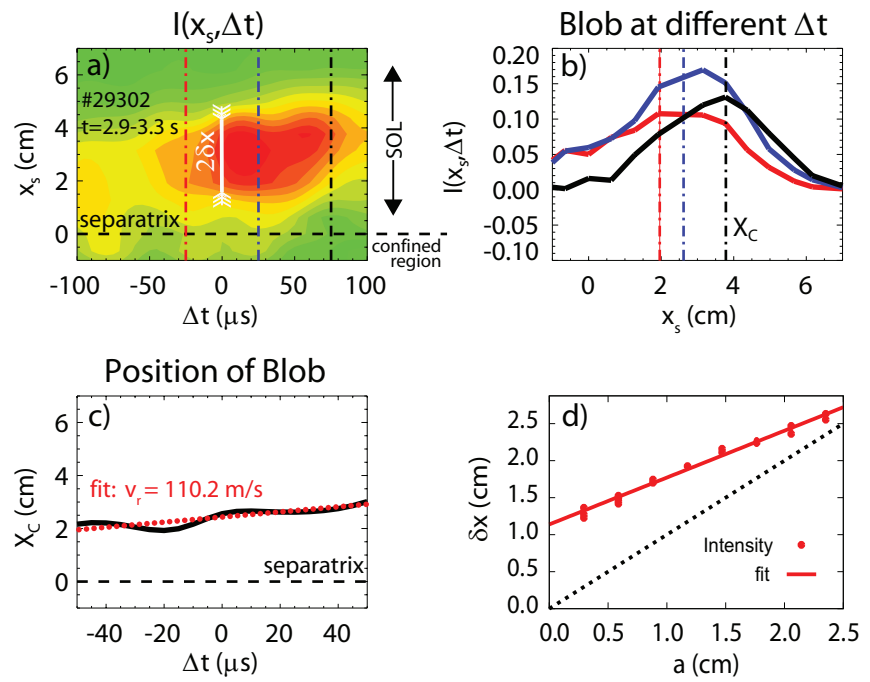

FIG. 3: (a) Color coded representation of the radial-temporal data $I\left(x_{s}, \Delta t\right)$ resulting from conditional averaging (red color corresponds to high intensity). (b) Radial profiles of the intensity response at different $\Delta t$ and the corresponding center-ofmass position $X_{C}$ (dashed lines). The different colors indicate different $\Delta t$ shown as dashed lines in (a). (c) Temporal evolution of the center-of-mass position (solid) and linear fit for velocity estimation (dotted). (d) Relation between measured intensity width $\delta x$ and model predicted density blob width $a$. The solid line indicates a linear fit of the simulation data (circles) and exceeds the one-to-one relation $\delta x=a$ (dashed).

a relative amplitude $\delta n / n$ on a measured density profile. Then the line emission profile was calculated from the density profile including the blob, and by comparison of the perturbed emission profile with the unperturbed emission profile, which is known from the measurement, 

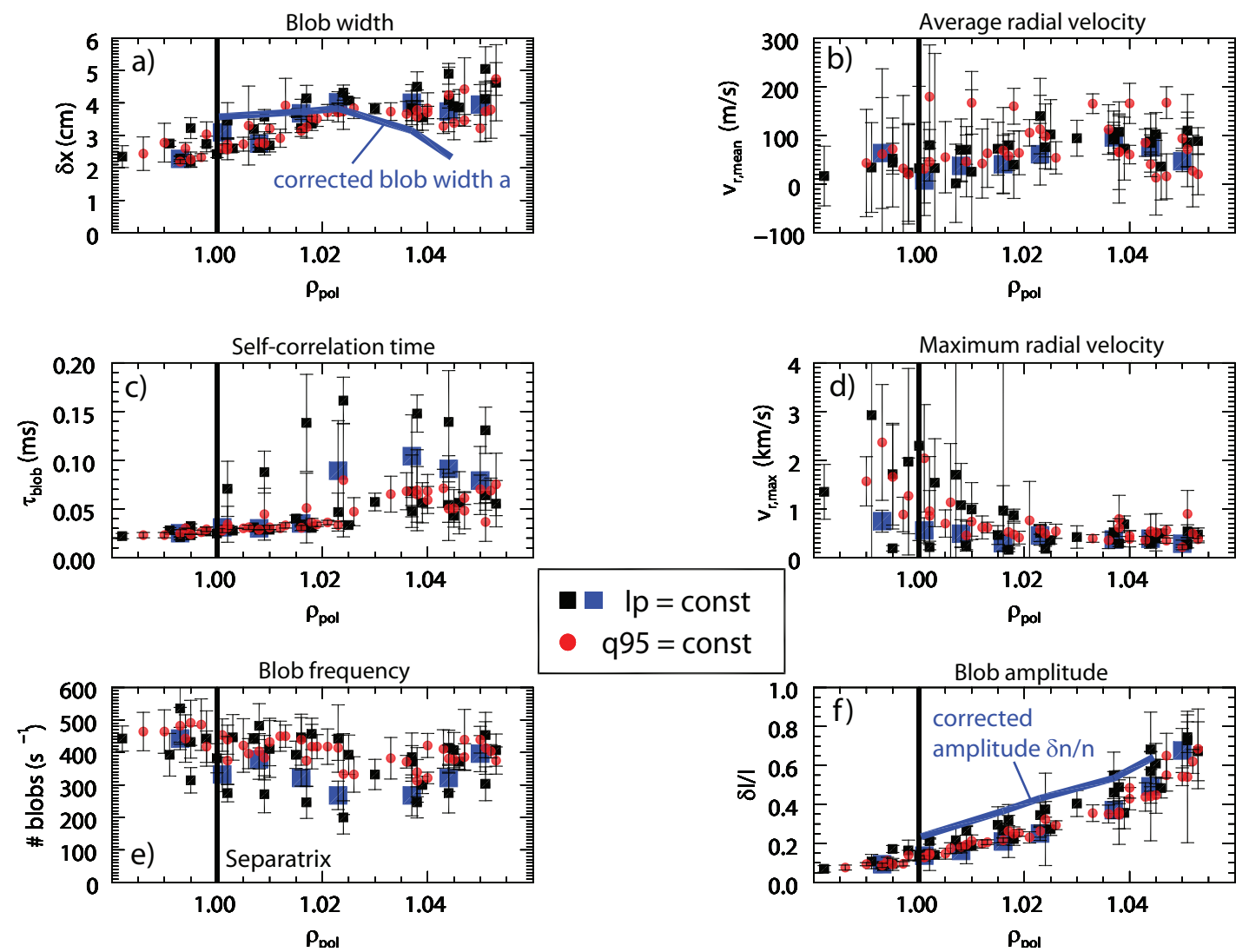

FIG. 4: Radial width of the blobs in the emission profile $\delta x(\mathrm{a})$, average radial blob velocity $v_{\mathrm{r}, \text { mean }}(\mathrm{b})$, self-correlation time $\tau_{\text {blob }}(\mathrm{c})$, maximum radial blob velocity $v_{\mathrm{r}, \max }(\mathrm{d})$, blob frequency (e), and relative intensity $\delta I / I$ (f) from conditional averaging depending on the radial position of the reference channel for discharges with constant $I_{p}$ (boxes) and $q_{95}$ (circles). The blue lines in (a) and (f) indicate the translated values $\delta x \rightarrow a$ and $d I / I \rightarrow d n / n$ for selected data points in discharge \#29302 (blue boxes).

the emission response of the blob $\delta x$ and $\delta I / I$ could be extracted. By variation of the input parameters $a, \delta n / n$ and the blob position, a translation of measured emission response quantities $\delta x$ and $\delta I / I$ to the input parameters $a$ and $\delta n / n$ is possible under realistic conditions. Since the relation between $\delta I / I$ and $\delta n / n$ is linear for blobs located in the far SOL, as shown in Fig. 6 of Ref. [12], and therefore easy to translate, we choose MSIG(6) corresponding to $\rho_{\text {pol }} \approx 1.044$ for quantitative comparisons with theory (see Section V). For this position, located 3 $\mathrm{cm}$ outside the separatrix in a distance of about $1.5 \mathrm{~cm}$ to the limiter, we obtained also a linear relation between $\delta x$ and $a$ from the numerical study as shown in Fig. 3(d).

\section{MAGNETIC FIELD DEPENDENCE OF BLOB PROPERTIES}

The tools described in Section III were used to analyze the data for the different discharges and time intervals given in Tab. I. Choosing different radial channels as reference for the conditional average procedure, we ob- tained blob characteristics as they appear in the lithium line emission at different radial positions. In order to identify general trends for the two data sets at constant $I_{p}$ (boxes) and $q_{95}$ (circles), respectively, Fig. 4 shows radial profiles of the parameters $\delta x(\mathrm{a}), v_{\mathrm{r}, \text { mean }}(\mathrm{b}), \tau_{\mathrm{blob}}$ (c), $v_{\mathrm{r}, \max }(\mathrm{d})$, the blob frequency (e) (definition provided below), and $\delta I / I$ (f) for all discharges given in Tab. I.

The emission response width $\delta x$ of the blobs is lowest $(\sim 2-3 \mathrm{~cm})$ near the separatrix and increases up to $4 \mathrm{~cm}$ at $\rho_{\text {pol }}=1.03$ for all discharge parameters. For larger radii, $\delta x$ saturates or further rises up to $5 \mathrm{~cm}$. This slight change of the behavior of $\delta x$ between the near $\operatorname{SOL}\left(\rho_{p o l}=1.00\right.$ to 1.03$)$ and far SOL $\left(\rho_{p o l}>1.03\right)$ is possibly related to the different density gradient lengths $L_{n}=1 / \nabla \ln n$ which is typically larger in the far SOL (cf. Fig. 1).

As mentioned above, the emission response width $\delta x$ is not identical to the "true" blob radius $a$. As exemplarily shown for discharge \#29302, the emission response $\delta x$ (blue boxes) underestimates the blob sizes a (blue line in Fig. 4(a)) for radii $\rho_{\text {pol }}<1.02$ (due to dominant collisional de-excitation and ionization of the $\mathrm{Li}_{2 \mathrm{p}}$ state) 
and overestimates $a$ for $\rho_{p o l}>1.02$ due to the "smearing" caused by the finite self-correlation time of the $\mathrm{Li}_{2 \mathrm{p}}$ state. The corrected blob width $a$ rises for $\rho_{p o l}<1.03$ and then decrease further outside.

The separation into two regions of different trends is also found in other parameters: the self-correlation time (Fig. 4(c)) increases with radius in the near SOL and decreases in the far SOL, while the blob frequency (Fig. $4(\mathrm{e})$ ) shows the opposite trend (falling frequency in the near SOL and rising in the far SOL). These two regions are possibly related to the radial dependence of the parallel connection length $L_{\|}$which falls below $15 \mathrm{~m}$ in the far SOL changing $L_{||}$-dependent blob properties and giving rise to a poloidal velocity shear layer as discussed in Ref. [19]. The maximum velocity $v_{\mathrm{r}, \max }$ (Fig. 4(d)) shows larger scatter $\left(v_{\mathrm{r}, \max } \sim 200-3000 \mathrm{~m} / \mathrm{s}\right)$ in the near SOL compared to the far SOL $\left(v_{\mathrm{r}, \max } \sim 200-1000 \mathrm{~m} / \mathrm{s}\right)$, and the slope of the radial dependence of $\delta I / I$ (Fig. 4(f)) seems slightly increased in the far SOL. An increase with radius is also found for the relative density amplitude $d n / n$ (blue line in Fig. 4(f)) determined from $d I / I$ (blue boxes) by means of the radiative-collisional model [12] for discharge \#29302.

The average velocity $v_{\mathrm{r} \text {,mean }}$ (Fig. $4(\mathrm{~b})$ ) varies for different discharges from $v_{\mathrm{r} \text {,mean }} \approx 10$ to $200 \mathrm{~m} / \mathrm{s}$ but shows almost no radial dependence.

In general, the blobs seem to grow with radius as it is evident in the self-correlation time $\tau_{b l o b}$ as well as in the relative amplitudes $\delta I / I$, and the radial dependences in the near SOL slightly differ from the far SOL.

In order to identify the relevant discharge parameters for the modification of the blob dynamics, we show how macroscopic parameters correlate with the blob activity in the far SOL at $\rho_{p o l}=1.044$. As a measure for the blob activity we take the number of events per second which surpassed the $2.5 \sigma$-threshold referred to as blob frequency. The result is shown in Fig. 5. Each symbol corresponds to a single discharge in the magnetic field scan at constant plasma current $I_{p}$ (boxes) and constant $q_{95}$ (circles). The error bars are the statistical variations (standard deviation) of the number of blobs per second determined in several subseries with a length of $56 \mathrm{~ms}$ each.

Figure 5(a) shows the blob frequency depending on the toroidal magnetic field $B_{t}$. While the blob frequency decreases for higher $B_{t}$ in the $I_{p}=$ const. case, it stays on the same level for constant $q_{95}$. Thus, the magnetic field cannot be the only parameter determining the blob frequency. The same conclusion can be drawn for the plasma current $I_{p}$ (Fig. 5(d)) which shows no correlation with blob frequency. A clear correlation, however, is seen in Fig. 5(c) where the blob frequency is strongly correlated with the edge safety factor $q_{95}$. This indicates a dependence of the blob frequency on the parallel connection length $L_{\|}$which scales proportional to the product $q_{95} R$ with major radius $R$.

It is assumed that the linear growth rate for curvature driven blobs is proportional to the normalized pressure

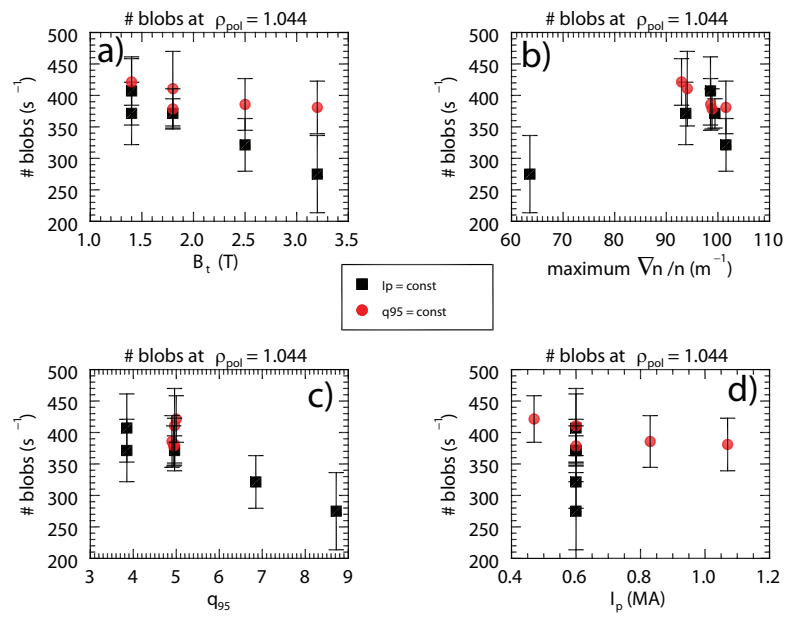

FIG. 5: Number of blobs per second depending on toroidal magnetic field $B_{t}(\mathrm{a})$, local density gradient at $\rho_{\text {pol }}=1.044$ (b), edge safety factor $q_{95}$ (c) and toroidal current $I_{p}$ (d) for different discharges with constant $I_{p}$ (boxes) or constant $q_{95}$ (circles).

gradient $\nabla p / p$ [2]. If the temperature profile is almost constant as in our case, it scales with the normalized density gradient $\nabla n / n$. In Fig. 5 (b) it is shown that the blob frequency is practically independent of the maximum normalized density gradient (typically found near the separatrix). Furthermore, Fig. 5(b) shows that the normalized gradients in the considered discharges have been kept at similar values around $100 \mathrm{~m}^{-1}$ (with one exception) suggesting a negligible systematic influence of the blob curvature drive on the dynamics discussed below.

As mentioned before, the sizes of the blobs are expected to decrease with increasing magnetic field as it was found in other experiments (e.g. [3, 20]). Furthermore, as discussed in theoretical works [4], it exists a most stable intermediate poloidal blob size since too small blobs decay due to the Kelvin-Helmholtz instability and too large blobs decompose into smaller interchange driven Rayleigh-Taylor fingers [21]. This intermediate blob size

$$
a^{*}=\rho_{s}^{4 / 5} L_{\|}^{2 / 5} / R^{1 / 5} \propto B^{-4 / 5}
$$

is also used for normalization of the governing equations of blob motion and can be interpreted as a scaling for the blob size. It is valid only if sheath resistivity as well as ion polarization drift effects regulate the blob dynamics. If finite ion temperature effects play a role, the scaling stays the same but with an additional prefactor $\sqrt[5]{8\left(1+\tau_{i}\right)}$ (see below and Ref. [11]).

In contrast to this blob size scaling, our measured radial blob sizes $a$ (not to be confused with the blob response width $\delta x$ ) in Fig. 6(a) do not clearly show the magnetic field dependence as expected from Eq. 4: Even for discharges with constant $q_{95}$ (corresponding to 

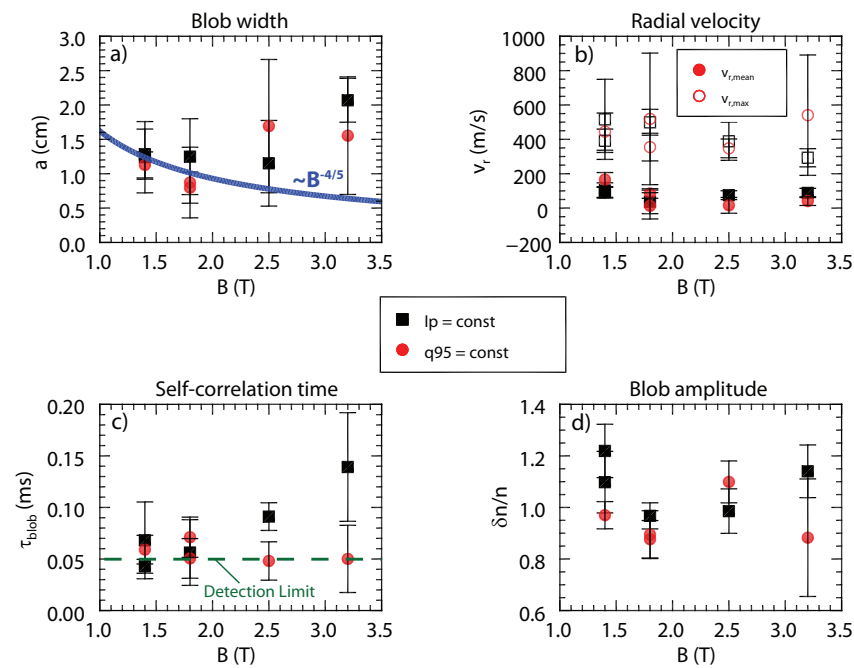

FIG. 6: Radial blob width $a$ (a), maximum and average radial blob velocity $v_{\mathrm{r}, \max }$ and $v_{\mathrm{r} \text {,mean }}(\mathrm{b})$, self-correlation time $\tau_{\text {blob }}(\mathrm{c})$ and relative blob amplitude $\delta n / n(\mathrm{~d})$ depending on toroidal magnetic field $B_{t}$. The blob width does not fit properly to the expexted $B^{-4 / 5}$-scaling (blue line).

constant $L_{\|}$), the expected dependence proportional to $B^{-4 / 5}$ (blue line) underestimates the measured values at higher fields albeit the error bars are large and the temperature dependence of $\rho_{s}$ could account for the deviations. For both data sets (constant $q_{95}$, circles, or constant $I_{p}$, boxes), the blobs have a similar size in the order of $1 \mathrm{~cm}$ despite the magnetic field was changed for more than a factor of two.

The radial blob velocity $v_{r}$ (Fig. 6(b)) exhibits a weak magnetic field dependence, too: The blobs tend to propagate faster at lower magnetic field as it is observed for the average velocities $v_{\mathrm{r} \text {,mean }}$ which amount to $v_{\mathrm{r} \text {,mean }} \approx 100$ $\mathrm{m} / \mathrm{s}$ at lower fields and decreasing at higher fields to $v_{\mathrm{r}, \text { mean }} \approx 50 \mathrm{~m} / \mathrm{s}$. The maximum velocities $v_{\mathrm{r}, \max }$ do not show a clear magnetic field dependence within the given error bars. The measured velocities will be related to the blob size in order to compare this relation with theoretical scaling laws (see Section V) which involve also other parameters than the magnetic field only.

While the blob size and the velocity scale not at all or only weakly with the magnetic field, the self-correlation time of the blobs seemingly exhibits a much clearer dependence on the magnetic field at constant plasma current $I_{p}$ (Fig. 6(c), squares). The measured selfcorrelation times $\tau_{\text {blob }}$ are higher than the lower limit of $50 \mu$ s given by the cut-off frequency of the filtering applied prior to the conditional averaging (cf. Section III), and seem to increase almost linearly with magnetic field from $\sim 50 \mu$ s up to $140 \mu \mathrm{s}$.

The data set at constant $q_{95}$ (Fig. 6(c), circles), however, shows almost no magnetic field dependence, which indicates that the $B_{t}$ dependence for the data set at constant $I_{p}$ is in fact a consequence of the $q_{95}$ dependence of the self-correlation time. Thus, the self-correlation time increases with $q_{95}$ and is hence determined by $L_{\|}$ only. This points to an impact of parallel losses scaling with $c_{s} / L_{\|}$on the self-correlation time since the (radial) velocities, which also could affect $\tau_{\text {blob }}$, are practically independent of $L_{\|}$.

Similar to the blob size $a$, the relative blob amplitude $\delta n / n$ exhibits no clear dependence on $B_{t}$ as shown in Fig. $6(d)$. Hence, the blob frequency (at least for constant $q_{95}$, cf. Fig. 5(a)), size $a$ and relative amplitude $\delta n / n$ featured no clear dependence on the magnetic field.

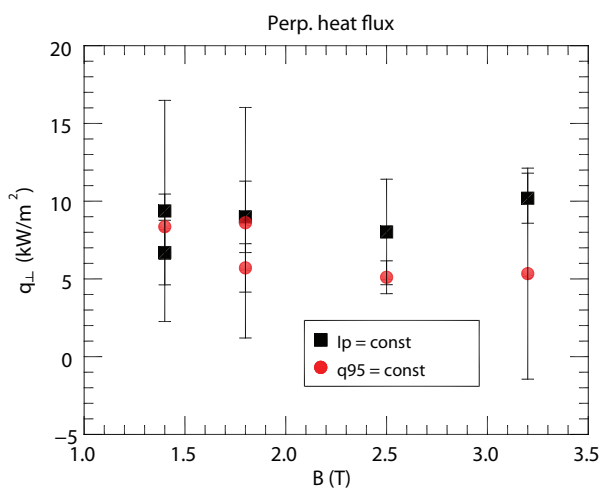

FIG. 7: Peak ion heat flux of a single blob depending on toroidal magnetic field $B_{t}$.

As a consequence, the peak ion heat flux carried by a single blob, $q_{\perp, i}=T_{i} \delta n v_{r}$, likewise shows no strong dependence on the magnetic field as shown in Fig. 7. $q_{\perp, i}$ is calculated assuming an ion temperature of $T_{i}=100 \mathrm{eV}$ as measured in filaments with a retarding field analyzer in L-mode [10]. The radial velocity is set to $v_{r}=v_{\mathrm{r}, \max }$ as an upper limit, and the measured $\delta n$ are in the range of $\delta n \approx 0.6$ to $2.0 \cdot 10^{18} \mathrm{~m}^{-3}$. Therefore, the peak ion heat flux of a blob amounts to several $\mathrm{kW} / \mathrm{m}^{2}$ and is therefore orders of magnitudes smaller than the parallel heat flux which is in the range of several $\mathrm{MW} / \mathrm{m}^{2}$ as measured with thermography on divertor target plates in the considered discharges.

\section{SCALINGS AND COMPARISON TO THEORY}

The measured blob velocities presented in Section IV are now compared to predictions from different theoretical velocity scaling laws on the basis of measured quantities like the blob size $a$, relative fluctuation level $\delta n / n$ or the connection length $L_{\|}$. We focus here on three velocity scaling laws which have in common that they assume a magnetic curvature driven vertical charge separation leading to an electric dipole around the density blob. This dipole is associated with a radial $E \times B$ drift moving the blob radially towards the wall at the outer mid plane. The three scaling laws differ in that they take 
into account different effects responsible for a reduction of the charge separation.

(i) The inertial scaling law [6] describes the charge separation to be dynamically balanced by perpendicular ion polarization currents leading to

$$
\frac{v_{r}}{c_{s}}=\sqrt{\frac{2 a}{R} \frac{\delta n}{n}},
$$

with $c_{s}=\sqrt{T_{e} / m_{i}}$ the sound velocity. Larger blobs are faster according to this scaling since they are less prone to be balanced by the polarization drift.

(ii) The sheath-connected scaling [4, 5]. Here, the charge separation is balanced by currents parallel to the magnetic field lines which form a closed circuit together with the sheath at the plasma facing component connected to the blob. The only limitation of the parallel currents in this model is the resistivity of the sheath resulting in the following scaling:

$$
\frac{v_{r}}{c_{s}}=2\left(\frac{\rho_{s}}{\delta_{b}}\right)^{2} \frac{L_{\|}}{R} \frac{\delta n}{n}=2 \ln 2\left(\frac{\rho_{s}}{a}\right)^{2} \frac{L_{\|}}{R} \frac{\delta n}{n} .
$$

In Ref. [5], the blob width $\delta_{b}$ was defined as a parameter in a Gaussian-shaped density perturbation which is related to our HWHM of the blob $a=\sqrt{\ln 2} \delta_{b}$.

These two regimes have been unified by Theiler et al. [9] and the measured blob velocities in a low-temperature plasma were shown to lie mostly below the presented scalings.

(iii) For the sheath-connected regime (Eq. 6) a similar scaling law was recently derived taking into account the effects of warm ions [11]. The scaling formulas are extended by a term including $\tau_{i}=T_{i} / T_{e}$, which is the ion-to-electron temperature ratio, and the blob amplitude $\delta n / n$. The curvature drive in the vorticity equation is enhanced by finite ion temperatures, and the blob velocity therefore is increased. Hence, the warm ion sheathconnected velocity scaling is

$$
\frac{v_{r}}{c_{s}}=\left(1+\tau_{i}\right)\left(\frac{\rho_{s}}{a}\right)^{2} \frac{L_{\|}}{R} \frac{\delta n}{n} .
$$

Magnetic field line fanning and shear [22] was not considered in the scalings since these parameters are believed to be small due to the fact that the considered field lines are not connected to the X-point region but hit on targets above the outer divertor baffle. In addition, these parameters should not change much in the present discharges.

We also neglect collisional effects which should play a minor role due to the low densities in the considered discharges. Whether collisions play a role can be quantified by a parameter

$$
\Lambda=\left(\frac{m_{e}}{m_{i}}\right)^{1 / 2} \frac{L_{\|}}{\lambda_{e}}
$$

with the mean free path of the electrons $\lambda_{e}$ as proposed by Myra et al. [7, 8]. Since $\Lambda<0.3$ at $\rho_{\text {pol }}=1.044$ for the considered discharges, collisional regime scaling formulas do not apply.

First we compare the measured blob sizes with theoretical most stable blob sizes. For cold ions, the most stable blob size $a^{*}$ obeys Eq. 4. But in the warm ion case, it is increased by a factor $\sqrt[5]{8\left(1+\tau_{i}\right)}[11]$. For the discharges given in Tab. I we had no direct measurements of the ion temperature in the SOL. But from comparable discharges we know that $\tau_{i} \approx 3$ at the considered radial position of $\rho_{\text {pol }}=1.044$ [10]. In Fig. 8(a), the measured blob sizes $a$ are compared with $a^{*}$ for the cold (triangles) and warm (diamonds) ion cases. As observed in many other experiments, the measured blob sizes exceed the most stable blob size for cold ions [2] in average by more than a factor of two. If the prefactor for warm ions is included into $a^{*}$, however, the measured blob sizes agree much better with the expectations. This is an indication that finite ion temperature effects play a significant role in the blob dynamics and hence for SOL transport.

In Fig. 8(b) the measured radial blob velocity $v_{r}$ depending on the measured blob size $a$ is displayed. For better comparison with theory, $v_{r}$ was normalized to the sound velocity $c_{s}$, and $a$ normalized to the drift scale $\rho_{s}$. Although the measured blob sizes $a$ had almost the same size (cf. Fig. 6(a)) at different $B_{t}$, the variation of the normalized blob size $a / \rho_{s}$ is large due to the magnetic field dependence of $\rho_{s}$. Both the average velocities $v_{\mathrm{r}, \text { mean }}$ (boxes) as well as the maximum velocities $v_{\mathrm{r}, \max }$ (circles) are highest for small normalized blob sizes and rather decrease for larger $a / \rho_{s}$. This is already a first contradiction to the inertial scaling (Eq. 5) which suggests higher velocities for larger blobs. In units of $c_{s}$, the measured average velocities range from $v_{\mathrm{r}, \text { mean }} / c_{s}=0.001$ to 0.0075 and are about five to ten times lower than the maximum velocities $v_{\mathrm{r}, \max } / c_{s}=0.012$ to 0.023 .

If we now assume a circular cross section of the blobs with radius $a$ in the plane perpendicular to the magnetic field, we can use our measured radial blob sizes $a$ for the scaling formulas which are derived for vertical blob sizes. Inserting $a, \delta n / n$, and the radial detection position $R=2.155 \mathrm{~m}$ into the inertial scaling law Eq. 5, we obtain velocities in the range of $v_{r} / c_{s} \approx 0.1$ which is more than an order of magnitude larger than the measured velocities. Together with the fact that the measured velocities rather decrease with increasing blob size in contrast to the scaling, we exclude a blob dynamics dominated by inertial effects for the considered data set. This is supported by the measured blob sizes which tend to be larger than $a^{*}$ resulting in a minor contribution of inertial effects $[9,21]$.

The velocities calculated from the sheath-connected formula according to Eq. 6 are shown in Fig. 8(b) (triangles). In addition to $a$ and $\rho_{s}$, the scaling formula depends also on $L_{\|}$given in Tab. I, which is the shortest parallel connection of the measurement position at the outer mid plane to the wall. Despite the variations of $L_{\|}$in different discharges, a reduced velocity with increasing size is clearly found. The absolute values from 

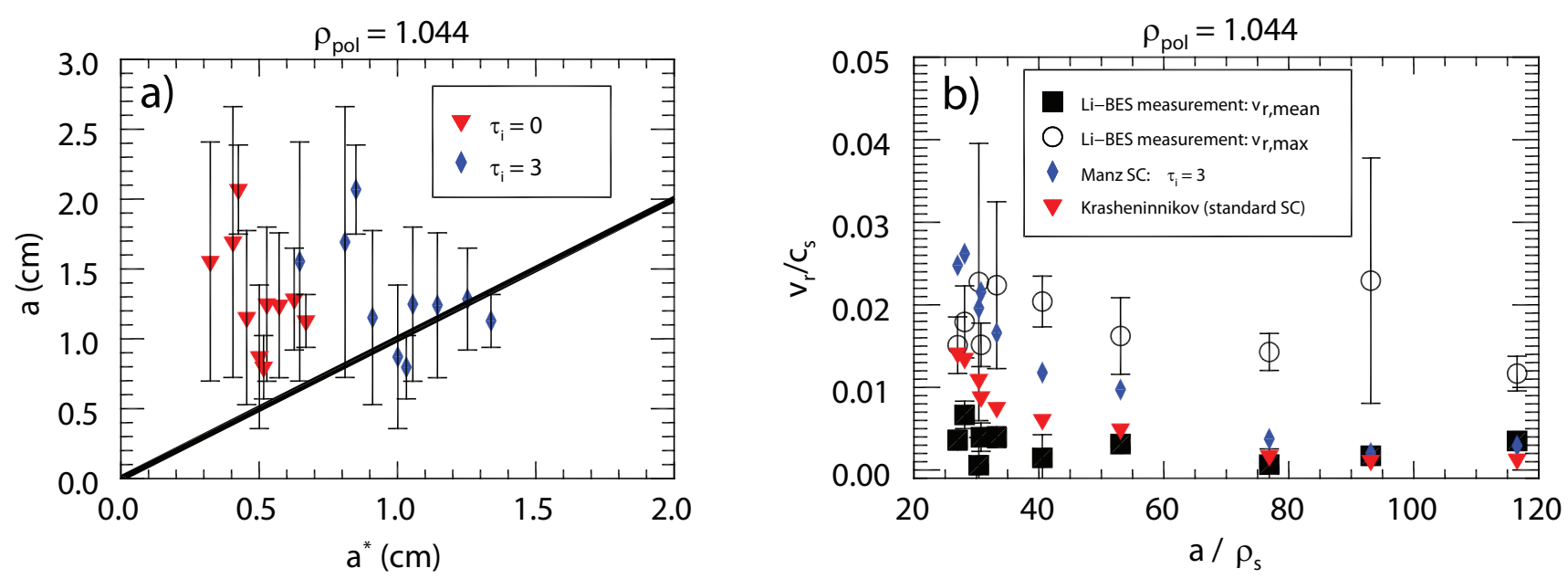

FIG. 8: (a) Measured blob size $a$ vs. most stable blob size $a^{*}$ with cold ions (triangles) and warm ion contribution, $\tau_{i}=3$ (diamonds). The measured blob sizes seem to be dominated by warm ion contributions. (b) Normalized measured blob velocities $v_{r} / c_{s}$ depending on normalized measured blob sizes $a / \rho_{s}$ (boxes: average velocity, circles: maximum velocities). Calculated velocities according to different scalings are added for comparison. While the sheath-connected regime formulas for cold (triangles) and warm (diamonds) ions exceed the average velocities $v_{\mathrm{r} \text {,mean }}$ for $a / \rho_{s}<40$, the maximum velocities $v_{\mathrm{r} \text {,max }}$ fit quite well to the scalings. For $a / \rho_{s}>70$, the scalings fit better to $v_{\mathrm{r}, \text { mean }}$.

the scaling formula agree well with the measured average velocities $v_{\mathrm{r}, \text { mean }}$ for larger blob sizes $\left(a / \rho_{s}>50\right)$. This is remarkable since rough approximations entered into the derivation of the scaling laws, and it shows the ability of the scalings to reasonably estimate the orderof-magnitude of blob velocities at a given blob size.

The measured maximum velocities $v_{\mathrm{r}, \text { max }}$ generally exceed the cold ion sheath-connected scaling velocities especially for large blob sizes. For smallest blob sizes $a / \rho_{s} \approx 30$, however, the scaling agrees well with the maximum velocities.

Taking warm ion effects into account with $\tau_{i}=3$ as it should be done in view of measured ion temperatures of about $30 \mathrm{eV}$ [10], the velocities in the warm ion sheathconnected regime according to Eq. 7 are too large compared with the average velocities (Fig. 8(b), diamonds). Only for largest blob sizes $\left(a / \rho_{s}>70\right)$, an agreement is found.

For small blob sizes $a / \rho_{s}<40$, the warm ion scaling fits very well to the measured maximum velocities $v_{\mathrm{r}, \max }$. In general, the measured values of $v_{\mathrm{r}, \max }$ fit better to the warm ion scaling than to the cold ion case. Since the scaling formulas should be related to the maximum velocities $v_{\mathrm{r}, \max }$ rather than to the average velocities $v_{\mathrm{r}, \text { mean }}$ as argued in [6], the better agreement of the warm ion case with the measurements points again to a possible influence of finite ion temperatures on the blob dynamics.

\section{SUMMARY AND CONCLUSION}

We have demonstrated that the Li-BES diagnostic is suitable to detect blobs and to quantify their spatiotemporal properties in the SOL of ASDEX Upgrade, if a careful data interpretation by means of a radiativecollisional modeling is applied. In low-density discharges, in which the SOL is in a sheath-connected regime (no divertor detachment, low collisionality), we found a strong reduction of the blob frequency with higher edge safety factor $q_{95} \sim L_{\|}$indicating the parallel transport time scale $\tau_{\|} \sim c_{s} / L_{\|}$to be involved in the blob generation mechanism.

The self-correlation time $\tau_{\text {blob }}$ likewise exhibits a strong $q_{95}$ dependence. Due to the impact of both the blob velocity and the intrinsic turbulent time scale on the measured self-correlation time, however, it is not easy to reveal the origin of the $q_{95}$ dependence.

The blob width seems to be only weakly dependent on $B_{t}$, which is in contradiction to the expected blob width scaling $a^{*}$ (Eq. 4). A similar result was obtained in the SOL of Alcator C-Mod and gyro-fluid simulations [23]. The blob velocity $v_{r}$ rather decreases with higher $B_{t}$, but the peak blob ion heat transport $q_{\perp, i}$ did not show any dependence on magnetic field $B_{t}$, plasma current $I_{p}$ or safety factor $q_{95}$.

The most remarkable result of our investigations is the good agreement of the measured blob sizes and velocities with the predictions from the scaling laws. Despite the fact that the scaling laws are only rough analytical simplifications derived from complex coupled equations, they predicted the correct order of magnitude of the sizes and velocities. This way, we could indirectly deduce that the blob dynamics in the considered discharges are dominated by the sheath-resistivity, since other scalings are not applicable at the given plasma conditions or they predicted too high magnitudes and wrong blob size dependencies of the velocity. The fact that the warm ion scaling formulas for the size and velocity fit better to the 
data than the cold ion scalings, indicates that warm ions contribute to size and propagation of the blobs. The latter point will be addressed in future investigations in different plasma regimes (collisional, electro-magnetic) and compared to gyro-fluid simulations in order to quantify the role of warm ions for perpendicular heat transport and their impact on plasma facing components in the main chamber wall.

\section{Acknowledgment}

This work, supported by the European Commission under the Contract of Association between EURATOM and ÖAW, was carried out within the framework of the European Fusion Development Agreement (EFDA). The views and opinions expressed herein do not necessarily reflect those of the European Commission.
[1] B. Lipschultz, X. Bonnin, G. Counsell, A. Kallenbach, A. Kukushkin, K. Krieger, A. Leonard, A. Loarte, R. Neu, R. Pitts, T. Rognlien, J. Roth, C. Skinner, J. Terry, E. Tsitrone, D. Whyte, S. Zweben, N. Asakura, D. Coster, R. Doerner, R. Dux, G. Federici, M. Fenstermacher, W. Fundamenski, P. Ghendrih, A. Herrmann, J. Hu, S. Krasheninnikov, G. Kirnev, A. Kreter, V. Kurnaev, B. LaBombard, S. Lisgo, T. Nakano, N. Ohno, H. Pacher, J. Paley, Y. Pan, G. Pautasso, V. Philipps, V. Rohde, D. Rudakov, P. Stangeby, S. Takamura, T. Tanabe, Y. Yang, and S. Zhu, Nuclear Fusion 47, 1189 (2007).

[2] D. A. D'Ippolito, J. R. Myra, and S. J. Zweben, Physics of Plasmas 18, 060501 (2011).

[3] S. J. Zweben, J. Boedo, O. Grulke, C. Hidalgo, B. LaBombard, R. J. Maqueda, P. Scarin, and J. L. Terry, Plasma Phys. Controll. Fusion 49, S1 (2007).

[4] S. I. Krasheninnikov, D. A. D'Ippolito, and J. R. Myra, Journal of Plasma Physics 74, 679 (2008).

[5] S. Krasheninnikov, Physics Letters A 283, 368 (2001).

[6] O. E. Garcia, N. H. Bian, and W. Fundamenski, Physics of Plasmas 13, 082309 (2006).

[7] J. R. Myra, D. A. Russell, and D. A. D'Ippolito, Physics of Plasmas 13, 112502 (2006).

[8] D. A. D'Ippolito and J. R. Myra, Physics of Plasmas 13, 062503 (2006).

[9] C. Theiler, I. Furno, P. Ricci, A. Fasoli, B. Labit, S. H. Müller, and G. Plyushchev, Phys. Rev. Lett. 103, 065001 (2009).

[10] M. Kočan, F. P. Gennrich, A. Kendl, H. W. Müller, and the ASDEX Upgrade Team, Plasma Physics and Controlled Fusion 54, 085009 (2012).

[11] P. Manz, D. Carralero, G. Birkenmeier, H. W. Müller, S. H. Müller, G. Fuchert, B. D. Scott, and U. Stroth, Physics of Plasmas (1994-present) 20, 102307 (2013).

[12] M. Willensdorfer, G. Birkenmeier, R. Fischer, F. M. Laggner, E. Wolfrum, G. Veres, F. Aumayr, D. Carralero, L.
Guimarais, B. Kurzan, and the ASDEX Upgrade Team, Plasma Physics and Controlled Fusion 56, 025008 (2014).

[13] S. J. Zweben, R. J. Maqueda, D. P. Stotler, A. Keesee, J. Boedo, C. E. Bush, S. M. Kaye, B. LeBlanc, J. L. Lowrance, V. J. Mastrocola, R. Maingi, N. Nishino, G. Renda, D. W. Swain, J. B. Wilgen, and the NSTX Team, Nucl. Fusion 44, 134 (2004).

[14] R. Fischer, E. Wolfrum, J. Schweinzer, and the ASDEX Upgrade Team, Plasma Physics and Controlled Fusion 50, 085009 (2008).

[15] B. Nold, G. D. Conway, T. Happel, H. W. Müller, M. Ramisch, V. Rohde, U. Stroth, and the ASDEX Upgrade Team, Plasma Physics and Controlled Fusion 52, 065005 (2010).

[16] F. Aumayr and H. Winter, Annalen der Physik 497, 228 (1985).

[17] M. Willensdorfer, E. Wolfrum, R. Fischer, J. Schweinzer, M. Sertoli, B. Sieglin, G. Veres, F. Aumayr, and the ASDEX Upgrade Team, Review of Scientific Instruments 83, 023501 (2012).

[18] H. Johnsen, H. L. Pécseli, and J. Trulsen, Phys. Fluids 30, 2239 (1987).

[19] B. Nold, P. Manz, T. T. Ribeiro, H. W. Mueller, G. Fuchert, G. Birkenmeier, M. Ramisch, B. D. Scott, U. Stroth, ASDEX Upgrade Team, submitted to New Journal Physics.

[20] G. Fuchert, G. Birkenmeier, B. Nold, M. Ramisch, and U. Stroth, Plasma Physics and Controlled Fusion 55, 125002 (2013).

[21] A. Y. Aydemir, Physics of Plasmas 12, 062503 (2005).

[22] D. A. Russell, J. R. Myra, and D. A. D'Ippolito, Physics of Plasmas 14, 102307 (2007).

[23] S. J. Zweben, B. D. Scott, J. L. Terry, B. LaBombard, J. W. Hughes, and D. P. Stotler, Physics of Plasmas 16, 082505 (2009). 